\title{
Can Ultrasound Be Used to Improve the Palpation Skills of Physicians in Training? A Prospective Study
}

\author{
Ryan Woods, MD, Steve J. Wisniewski, MD, Daniel R. Lueders, MD, \\ Thomas P. Pittelkow, DO, MPH, Dirk R. Larson, MS, Jonathan T. Finnoff, DO
}

\begin{abstract}
Background: Accurate diagnosis of musculoskeletal disorders relies heavily on the physical examination, including accurate palpation of musculoskeletal structures. The literature suggests that there has been a deterioration of physical examination skills among medical students and residents, in part due to increased reliance on advanced imaging. It has been shown that knowledge of musculoskeletal anatomy and physical examination skills improve with the use of ultrasound; however, the literature is limited. Objective: To determine whether ultrasound can improve the ability of physicians in training (residents) to palpate the long head of the biceps tendon (LHBT) in the bicipital groove.
\end{abstract}

Design: Prospective study design.

Setting: Tertiary care center.

Participants: Ten physical medicine and rehabilitation residents served as subjects. Exclusion criteria included the presence of any condition that precluded their ability to palpate. Three volunteers were used as models. Model exclusion criteria included anything that distorted normal shoulder anatomy or inhibited examiner palpation. Three investigators with experience performing diagnostic musculoskeletal ultrasound were used to confirm palpation attempts.

Methods: Subjects attempted to palpate the LHBT bilaterally in the bicipital groove of each model. Investigators assessed the accuracy of the palpation attempt using real-time ultrasonography. Subjects participated in a 30-minute ultrasound-assisted training session learning how to palpate the LHBT in the bicipital groove with ultrasound confirmation. After the ultrasound training session, subjects again attempted to palpate the LHBT in the bicipital groove of each model with investigator confirmation.

Main Outcome Measurements: LHBT palpation accuracy rates preintervention versus postintervention.

Results: Pretraining LHBT palpation accuracy was $20 \%$ (12/60 attempts). Post-ultrasound training session accuracy was $51.7 \%$ (31/60 attempts; $P \leq .001)$.

Conclusions: Our findings demonstrate that palpation accuracy improves after ultrasound assisted LHBT palpation training. These data suggest that the use of ultrasound may be beneficial when teaching musculoskeletal palpation skills to health care professionals.

Level of Evidence: II

\section{Background and Significance}

Accurate diagnosis of musculoskeletal disorders relies heavily on the physical examination. A vital component of the musculoskeletal physical examination involves accurate palpation of musculoskeletal structures and knowledge of the underlying anatomy. By accurately identifying the painful structure, the differential diagnosis can be narrowed, and a more precise diagnosis can be made. Traditionally, surface landmarks have been used as a point of reference to guide palpation of anatomic structures and have been a mainstay in teaching the musculoskeletal examination. Physical examination palpation skills historically have been taught through classroom education, mentorguided bedside examination of patients, or through simulation models. The literature, however, suggests that physical examination skills have deteriorated among medical students and residents, and that this decline is in part due to increased reliance on advanced imaging and reduced supervised bedside examination teaching [1-3]. 
A study by Gazzillo et al [4] revealed that a physical medicine and rehabilitation (PM\&R) resident, sports medicine fellow, and a board-certified sports medicine physician were only able to correctly palpate the long head of the biceps tendon (LHBT) within the intertubercular groove $0 \%, 12 \%$, and $5.3 \%$ of the time, respectively [4]. Rho et al [5] demonstrated similar results, with PM\&R residents only able to accurately palpate the acromioclavicular (AC) joint and lateral knee joint line $16.7 \%$ and $58.3 \%$ of the time, respectively.

Interventional procedures that use surface landmark palpation to indentify structures are also susecptible to palpation-guided inaccuracies. Peck et al [6] performed a cadaveric study that demonstrated the AC joint was only able to be accurately injected $40 \%$ of the time via palpation-guidance. Scillia et al [7] confirmed similar results in an in vivo study. AC joint injections were done by experienced fellowship-trained musculoskeletal radiologists. Their results demonstrated that only $36.5 \%$ of the injections performed were confirmed to be in the AC joint when performed via a digital palpation technique. Similar accuracy studies have been performed with knee joint injections. Curtiss et al [8] performed a cadaveric study demonstrating that less-experienced clinicians were only able to accurately inject the knee joint $55 \%$ of the time using a superolateral approach. Wisniewski et al [9] also completed a cadaveric study comparing ultrasound-guided sinus tarsi injections with palpationguided sinus tarsi injections and found only a $35 \%$ accuracy rate via palpation guidance.

Certainly, there are challenges with intra-articular landmark-based palpation-assited interventions, but this also extends to large soft-tissue regions as well. Karvelas et al [10] performed a recent electromyography (EMG) study using ultrasound to confirm EMG needle placement into target muscles using traditional surface anatomy and landmark palpation as a guide. Their results demonstrated a mean accuracy of EMG needle placement into 4 specific muscles of the upper or lower extremities to be $50 \%$ and $82.1 \%$ for postgraduate year 3 and postgraduate year 4 residents, respectively [10]. The aforementioned studies confirm the fact that in many instances, physicians of all levels lack the ability to correctly identify musculoskeletal anatomy and perform accurate interventional procedures through the use of surface landmarks alone.

Musculoskeletal sonography in medical education is becoming more popular. Hoppman et al [11] demonstrated that after brief ultrasound training, fourth-year medical students were able to accurately identify knee joint effusions using ultrasound. Shapiro et al [12] demonstrated that basic diagnostic ultrasound skills could be taught to medical students in a relatively short time, with students showing a favorable response to using ultrasound as an adjunct to learning the physical examination. Furthermore, it has been shown that knowledge of musculoskeletal anatomy and physical examination skills improve with the use of ultrasound, although it is imperative for the learner to first have an understanding of the anatomy and physical examination to maximize the learning affect associated with ultrasound [13-15].

Although there is preliminary research suggesting ultrasound can assist with learning anatomy and physical examination palpation skills [16], the literature is still quite limited. Therefore, the primary aim of this study was to determine whether ultrasound could improve the ability of physicians in training (residents) to palpate the LHBT in the bicipital groove. We hypothesized that integrating ultrasound into the learning process would significantly improve their ability to accurately palpate the LHBT.

\section{Methods}

This study was completed in an outpatient tertiary care academic center and was approved by the institutional review board at the authors' institution. Ten current PM\&R residents within our institution were recruited by word of mouth to serve as subjects for the investigation. Exclusion criteria included the presence of peripheral neuropathy, peripheral vascular disease, peripheral inflammatory condition, active infection, or other condition that precluded their ability to palpate. The age, gender, and level of medical training (ie, year in residency) of each subject was recorded (Table 1).

Three models were recruited by word of mouth to participate in the study. Exclusion criteria for the models included individuals with current shoulder pain, previous shoulder surgery, or anatomic deformities or anomalies found during a preparticipation diagnostic ultrasound of the shoulder. This ensured normal shoulder anatomy in the models and eliminated clinical signs that may assist or inhibit the examiner during shoulder palpation. The models' height, weight, and calculated body mass index was recorded (Table 2). Transverse dimensions of the bicipital groove from the highest

Table 1

Subject demographics

\begin{tabular}{ll}
\hline Demographics & Total, N=10 \\
\hline Age & \\
Mean (SD) & $30.1(1.4)$ \\
Median & 30 \\
Range & $(28.0-33.0)$ \\
Gender & \\
Male & $5(50 \%)$ \\
Female & $5(50 \%)$ \\
Level of training & \\
PGY 2 & 2 \\
PGY 3 & 3 \\
PGY 4 & 5 \\
\hline
\end{tabular}

$\mathrm{PGY}=$ postgraduate year. 
Table 2

Model demographics

\begin{tabular}{ll}
\hline Demographics & Total, $\mathrm{n}=3$ \\
\hline Height, $\mathrm{cm}$ & \\
Mean (SD) & $174.4(5.3)$ \\
Median & 172.7 \\
Range & $(170.2-180.3)$ \\
Weight, kg & \\
Mean (SD) & $73.3(8.6)$ \\
Median & 77.1 \\
Range & $(63.5-79.4)$ \\
BMl, kg/m ${ }^{2}$ & \\
Mean (SD) & $24.1(2.7)$ \\
Median & 24.4 \\
Range & $(21.3-26.6)$ \\
\hline
\end{tabular}

$\mathrm{SD}=$ standard deviation; $\mathrm{BMI}=$ body mass index.

points of the lesser and greater tuberosities (millimeters) and the depth from the skin to the deepest aspect of the bicipital groove (millimeters) also were recorded for each model (Table 3). Informed consent was obtained from the subjects and models before participation in the study.

Each of the subjects had previously completed a musculoskeletal physical examination course as part of their residency training, where they learned how to palpate the LHBT in the bicipital groove using surface landmarks. The physical examination course is held yearly during the residency program, and thus residents had completed this course 1-3 times, depending on their level of training. All of the subjects were currently using these palpation skills to evaluate patients in a clinical setting.

On enrollment in the study, the subjects were asked to palpate the LHBT bilaterally in the bicipital groove of each model. Models were placed supine on a flat examination table with a small pillow under their head for comfort. The models were instructed to rest their

Table 3

Model bicipital groove anatomy

\begin{tabular}{ll}
\hline Measurement & Total, $\mathrm{n}=3$ \\
\hline Width of groove, left, mm & \\
Mean (SD) & $9.2(1.0)$ \\
Median & 9.1 \\
Range & $(8.3-10.3)$ \\
Width of groove, right, mm & \\
$\quad$ Mean (SD) & $9.2(1.5)$ \\
Median & 9.3 \\
Range & $(7.6-10.6)$ \\
Skin to deepest part of groove, left, mm & \\
$\quad$ Mean (SD) & $17.7(2.6)$ \\
Median & 18.7 \\
$\quad$ Range & $(14.8-19.6)$ \\
Skin to deepest part of groove, right, mm & \\
$\quad$ Mean (SD) & $17.7(2.9)$ \\
Median & 18.2 \\
Range & $(14.6-20.3)$ \\
\hline
\end{tabular}

$\mathrm{SD}=$ standard deviation. shoulders and arms on the table. The elbow of the arm being evaluated was then flexed $90^{\circ}$. An Acumar Digital Inclinometer (Acumar Technology, North Lafayette, IN), was placed on the ulnar aspect of the distal forearm, proximal to the ulnar styloid process, and secured to the forearm with tape. The inclinometer was then calibrated to "0," designating the starting position. Subjects then attempted to localize the right LHBT in the bicipital groove via palpation while internally and externally rotating the shoulder. The subject positioned the shoulder in internal or external rotation such that the LHBT was pointing directly anterior (towards the ceiling) (Figure 1). The subject then placed a standard stainless-steel, 18-gauge Tuohy needle on the anterior shoulder skin surface over the LHBT in plane (ie, colinear) with the tendon. The needle was secured to the skin by the use of transparent tape (Figure 2). A coinvestigator held the model's arm in position ensuring that it did not move by taking note of the inclinometer reading and keeping it constant (Figure 3 ). The subject then left the room.

Three investigators (R.R.W., D.R.L., T.P.P.) (1 investigator per model) with experience performing diagnostic musculoskeletal ultrasound assessed the accuracy of LHBT palpation using real-time ultrasonography. The investigators had 3 (R.R.W.), 4 (D.R.L.), and 5 (T.P.P.) years of experience using diagnostic musculoskeletal ultrasound, respectively. The sonographic evaluation was performed with a Samsung RS80A (Samsung Medison Co, Ltd, Seoul, South Korea) and 2 Philips CX50 ultrasound machines (Philips Ultrasound Systems, Bothell, WA). The aforementioned ultrasound machines have been approved by the Food and Drug Administration for indicated use. All ultrasound scans were performed with a 12-3 megahertz linear array transducer. A second Acumar Digital Inclinometer was taped to the

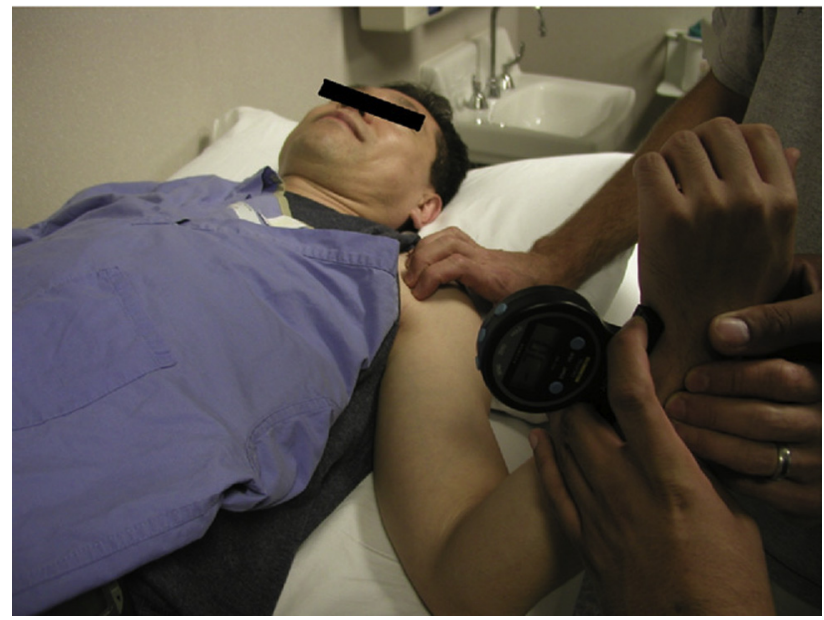

Figure 1. Subjects positioned the model's shoulder such that the long head of biceps tendon was pointing directly anterior. The subjects then attempted to palpate the long head of biceps tendon within the intertubercular groove. 


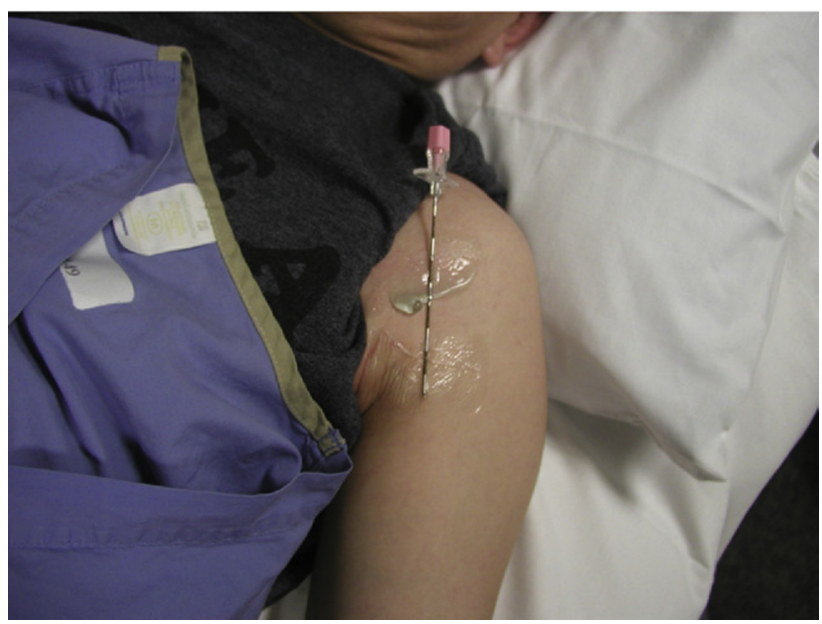

Figure 2. A needle was placed colinear to the presumed position of the long head of the biceps tendon and secured to the skin via the use of transparent tape.

transducer and calibrated with the transducer so that when zeroed, the head of the transducer was parallel to the floor.

Investigators placed the ultrasound transducer directly over the needle on the skin surface in a shortaxis relative to the needle and LHBT (Figure 4). The transducer was manipulated using "heel-toe" maneuvers to ensure the head of the transducer was parallel to the floor using the inclinometer. The needle was identified on the ultrasound screen due to its superficial location and hyperechoic echotexture. If the greater and lesser tuberosities were not readily apparent, the transducer was translated superiorly and inferiorly to identify them. When the needle, the LHBT, and the greater and lesser tuberosities were all visualized, an ultrasound image was saved. Once completed, the entire aforementioned process was repeated on the

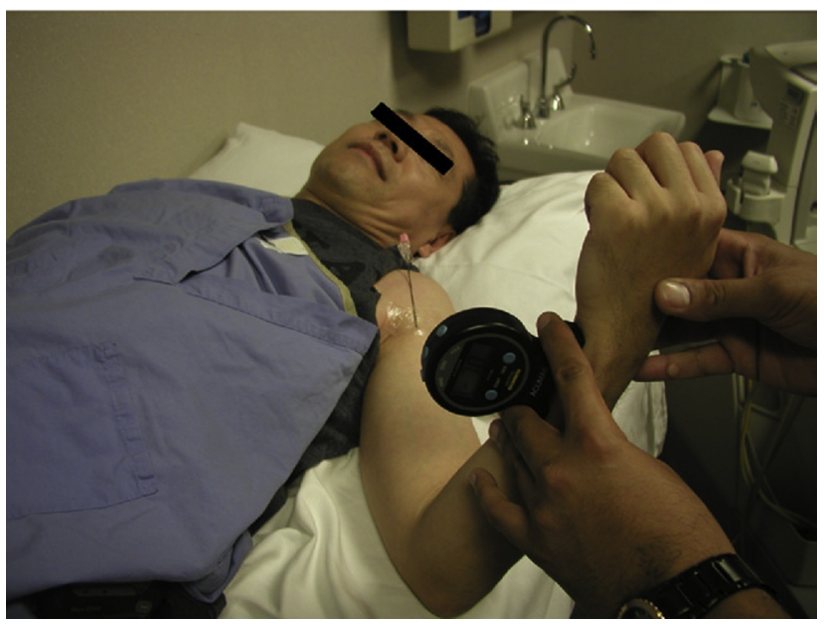

Figure 3. A coinvestigator held the model's arm in position ensuring that it did not move by taking note of the inclinometer reading and keeping it constant.

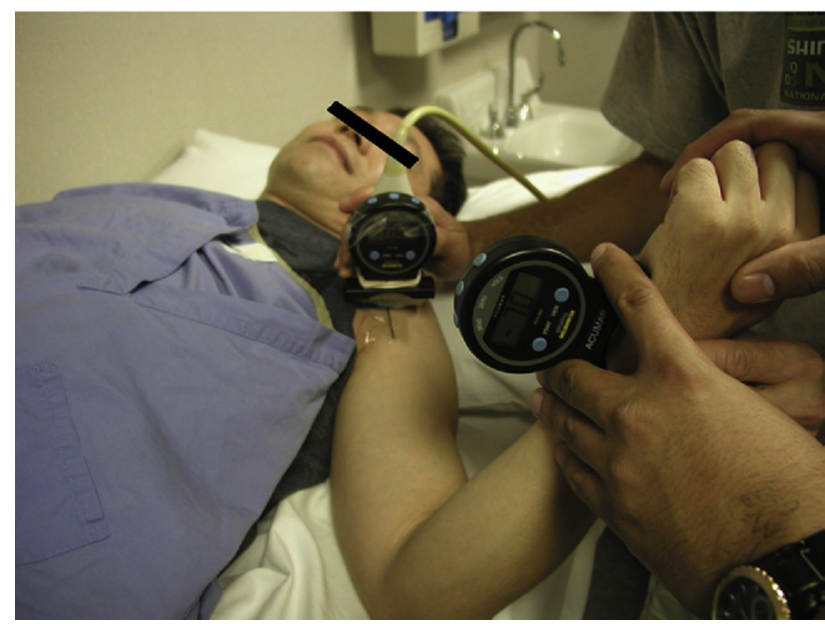

Figure 4. Investigators placed the ultrasound transducer directly over the needle on the skin surface in a short-axis relative to the needle and long head of biceps tendon.

contralateral (left) side by each subject and investigator on the same model. The subject then repeated this process on the other 2 models.

Subjects next participated in a 30-minute ultrasoundassisted training session to learn how to palpate the LHBT in the bicipital groove with ultrasound confirmation. During this training session, the subjects served as models for each other to practice palpating the LHBT in the bicipital groove. The training session was led by a PM\&R sports medicine staff physician with 10 years of musculoskeletal ultrasound experience. The same ultrasound machines previously described were used during this training session. The subjects palpated the LHBT in the bicipital groove and used the ultrasound imaging to provide immediate feedback regarding their accuracy. They practiced this repetitively on multiple subjects throughout the 30-minute session. They were also provided with access to anatomy books to assist with their knowledge of shoulder anatomy.

After completion of the ultrasound training session, subjects again attempted to palpate the LHBT in the bicipital groove of each model using the method previously described. Investigators again obtained and stored images of the palpation attempts as previously described.

After the subject's posttraining palpation attempt, investigators reviewed all images. The primary outcome of interest in this study was palpation accuracy of the LHBT within the bicipital groove. With each saved image, a T-square was placed on top of the ultrasound machine monitor to provide a vertical axis for measurements. The T-square was shifted left or right over the ultrasound monitor until its vertical edge passed directly through the needle of the stored images. The investigator recorded the presence or absence of the T-square line within the bicipital groove. An attempt was determined to be accurate when the vertical edge 
of the T-square passing through the needle landed between the peaks of greater and lesser tuberosities within the bicipital groove (Figure 5).

Secondary outcomes included distance and direction of the needle placement from the bicipital groove after a missed attempt. An attempt was determined to be a miss if the vertical edge of the T-square passing through the needle fell outside of bicipital groove, either medial to the peak of the lesser tuberosity or lateral to the peak of the greater tuberosity (Figures 6 and 7). If the T-square line passed outside the groove, the location (medial or lateral) and distance (millimeters from the $\mathrm{T}$-square line to the closest edge of the bicipital groove) were recorded. Negative numbers indicated medial placement, whereas positive numbers indicated a lateral placement from the bicipital groove.

The palpation accuracy of the pretraining session was then compared with the palpation accuracy of the posttraining session to determine whether there was a change in the ability to accurately palpate the LHBT after the ultrasound training session.

\section{Statistical Analysis}

All data were summarized by the use of descriptive statistics, including means and standard deviations for continuous data, and counts and percentages for categorical variables. The primary outcome was the accuracy of palpating the LHBT; this was reported and analyzed as (1) accurately palpating the LHBT in the bicipital groove (no/yes), (2) the direction of the subject-examiner's location and the actual location of the LHBT (medial, accurate, lateral), and (3) the distance (in millimeters) between the subject-examiner's location and the actual location of the LHBT. Because each subject examined each of the 3 human models, the

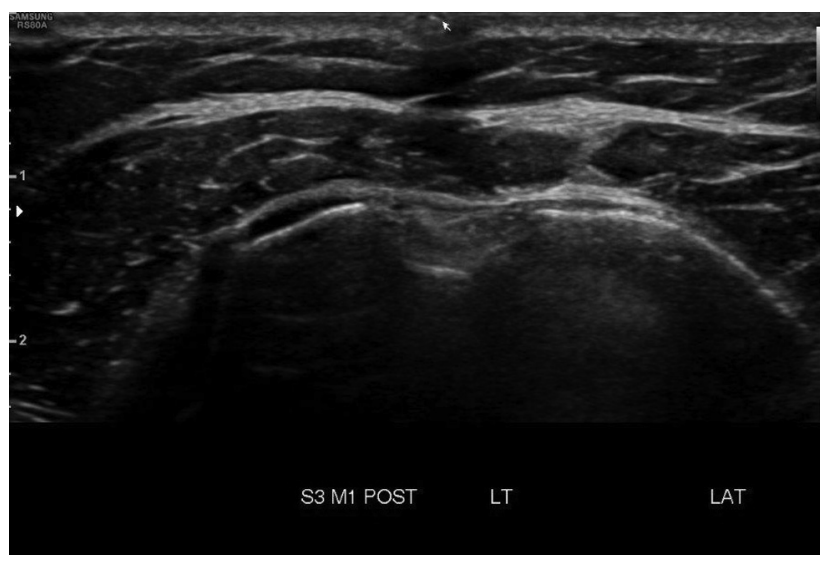

Figure 5. Sonographic image of a correct palpation attempt. The needle (arrowhead) is located directly over the intertubercular groove. S3 M1 POST, subject 3, model 1, post-ultrasound training; LT, left shoulder; LAT, lateral. Top of figure indicates superficial, left of figure indicates medial, right of figure indicates lateral, and bottom of figure indicates deep.

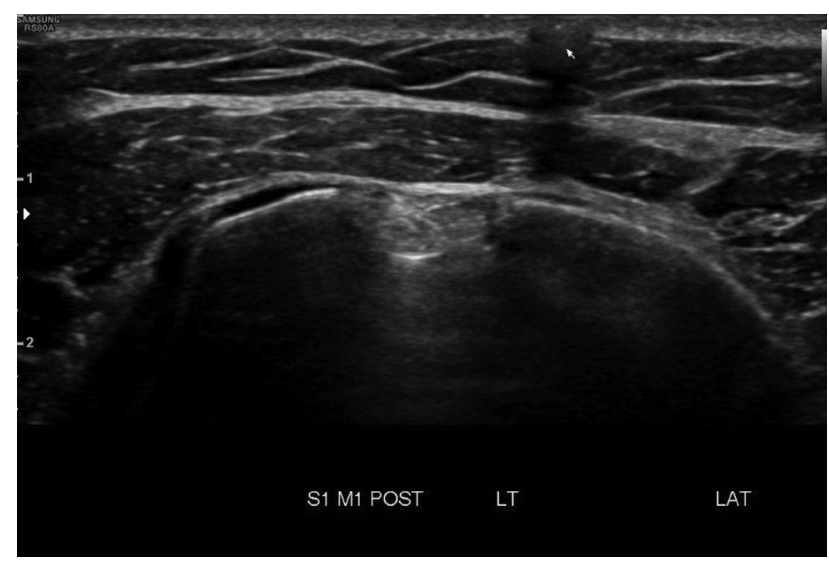

Figure 6. Sonographic image of a laterally missed palpation attempt. The needle (arrowhead) is located lateral to the intertubercular groove. S1 M1 POST, subject 1, model 1, post-ultrasound training; LT, left shoulder; LAT, lateral. Top of figure indicates superficial, left of figure indicates medial, right of figure indicates lateral, and bottom of figure indicates deep.

primary analysis was conducted using generalized linear models using generalized estimating equations to properly account for the within-subject correlation.

Using the generalized linear modeling framework described previously, we evaluated the association of the ultrasound training intervention and the accuracy of palpating the LHBT in the bicipital groove (no/yes) using logistic regression. Similarly, using the generalized linear modeling approach described previously, we analyzed the effect of the intervention and the distance between the subject examiner's attempt and the actual location of the LHBT (both actual and absolute) using linear regression. The association of the intervention and the directional outcome (medial, accurate, lateral) was analyzed with multinomial logistic regression (based on the generalized logit model) via a generalized

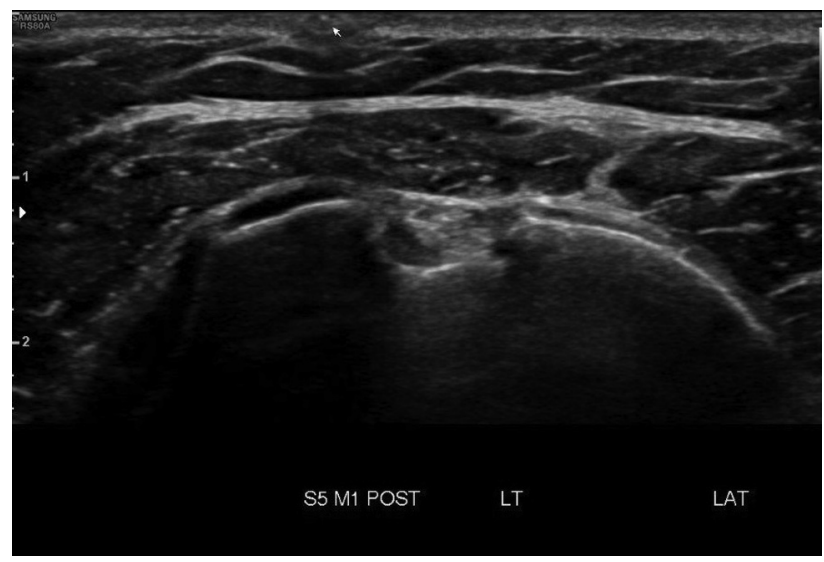

Figure 7. Sonographic image of a medially missed palpation attempt. The needle (arrowhead) is located medial to the intertubercular groove. S5 M1 POST, subject 5, model 1, post-ultrasound training; LT, left shoulder; LAT, lateral. Top of figure indicates superficial, left of figure indicates medial, right of figure indicates lateral, and bottom of figure indicates deep. 
liner mixed model with subjects included as a random effect to account for the within-subject correlation. Model results were reported using odds ratios and 95\% confidence intervals $(\mathrm{Cls})$ for the logistic and multinomial models, and parameter estimates corresponding to pre- versus postintervention differences for the linear models.

As a secondary analysis, the data were collapsed to the number of correct palpations per subject (out of 6 attempts: left and right side for each of 3 human models) before the ultrasound training and after the ultrasound training. In this analysis, the data comprised 10 independent observations (1 per subject corresponding to number of accurate attempts for that subject) for both the pre- and posttraining periods; the preultrasound training values were compared with the post-ultrasound values via a Wilcoxon signed-rank test. All statistical tests were 2 -sided, and $P$ values less than .05 were considered significant.

\section{Results}

Training levels of the subjects (PM\&R residents) varied from postgraduate year 2 to postgraduate year 4 . Resident age ranged from 28 to 33 years old. Male and female residents were equally represented. The subjects' demographic data are outlined in Table 1.

Two male and one female nonresident physicians were recruited as models. Mean height, weight, and body mass index were $174.4 \mathrm{~cm}, 73.3 \mathrm{~kg}$, and $24.1 \mathrm{~kg} / \mathrm{m}^{2}$, respectively. Bilateral bicipital groove height and width was recorded from each model. Mean bicipital groove width was $9.2 \mathrm{~mm}$ (7.6-10.6), with a mean depth of $17.7 \mathrm{~mm}$ (14.6-20.3). Demographic and anatomic data for the models are outlined in Tables 2 and 3.
Pretraining LHBT palpation accuracy was 20\% (12/60 attempts). Post-ultrasound training session accuracy was $51.7 \%$ (31/60 attempts); palpation attempts were 4.2 times more likely to be in the correct location after ultrasound training than at baseline $(95 \% \mathrm{Cl} 1.8-9.9, P<$ .001; Table 4).

Of the 60 pretraining attempts, 12 of $60(20 \%)$ were accurate, 22 of 60 (36.7\%) were missed medially, and 26 of $60(43.3 \%)$ were missed laterally. Of the 60 posttraining attempts, 31 of $60(51.7 \%)$ were accurate, 5 of 60 (8.3\%) were missed medially (odds ratio $0.08,95 \% \mathrm{Cl}$ $0.02-0.39, P=.002$ ), and 24 of $60(40 \%)$ were missed laterally (odds ratio $0.36,95 \% \mathrm{Cl} 0.11-1.12, P=.08$, Table 4). These results indicate that after the ultrasound training, subjects were 12.5 times less likely to miss in the medial direction and 2.8 times less likely to miss in the lateral direction compared with the pretraining palpation attempts. Distance away from the bicipital groove was measured either medially or laterally from the midpoint of the needle to the peak of the lesser and greater tuberosities respectively. The mean direction and distance missed pretraining was $0.9 \mathrm{~mm}$ medially with a range of $29.5 \mathrm{~mm}$ medially to $22.9 \mathrm{~mm}$ laterally. The mean posttraining direction and distance missed was $2.5 \mathrm{~mm}$ laterally with a range of $14.9 \mathrm{~mm}$ medially to $14.7 \mathrm{~mm}$ laterally. Thus, after the ultrasound training intervention, the mean distance and direction of the miss shifted $3.4 \mathrm{~mm}$ in the lateral direction $(95 \% \mathrm{Cl}$ 0.4-6.4, $P=.03)$. Absolute distance from the groove (ignoring direction) of pre- and posttraining missed attempts was $7.5 \mathrm{~mm}$ and $4.4 \mathrm{~mm}$, respectively. Thus, the accuracy improved by $3.1 \mathrm{~mm}$ (95\% $\mathrm{Cl} 0.5-5.7, P=.02)$ from the pretraining to posttraining periods. Accuracy rates, direction, and distance results are summarized in Table 4.

Table 4

Primary and secondary outcomes

\begin{tabular}{|c|c|c|c|c|}
\hline & \multicolumn{2}{|c|}{$(-)$ Medial $(+)$ Lateral } & \multirow{2}{*}{$\begin{array}{l}\text { Odds Ratio or Difference } \\
\text { in Means }(95 \% \mathrm{Cl})\end{array}$} & \multirow[b]{2}{*}{$P$ Value } \\
\hline & Pre & Post & & \\
\hline Within groove & $N=60$ & $\mathrm{~N}=60$ & & \\
\hline Yes & $12(20 \%)$ & $31(51.7 \%)$ & $4.2(1.8-9.9)^{*}$ & $<.001$ \\
\hline No & $48(80 \%)$ & $29(48.3 \%)$ & & \\
\hline \multicolumn{5}{|l|}{ Direction of miss } \\
\hline Within groove & $12(20 \%)$ & $31(51.7 \%)$ & & \\
\hline Medial & $22(36.7 \%)$ & $5(8.3 \%)$ & $0.08(0.02-0.39)^{\dagger}$ & .002 \\
\hline Lateral & $26(43.3 \%)$ & $24(40.0 \%)$ & $0.36(0.11-1.12)^{\dagger}$ & .08 \\
\hline \multicolumn{5}{|c|}{ Distance away from groove, $\mathrm{mm}$} \\
\hline Mean (SD) & $-0.9(10.7)$ & $+2.5(6.5)$ & $3.4(0.3-6.4)^{\ddagger}$ & .03 \\
\hline Median & 0 & 0 & & \\
\hline Range & -29.5 to 22.9 & -14.9 to 14.7 & & \\
\hline \multicolumn{5}{|c|}{ Absolute value of distance away from groove, $\mathrm{mm}$} \\
\hline Mean (SD) & $7.5(7.6)$ & $4.4(5.4)$ & $-3.1(-5.7 \text { to }-0.5)^{\ddagger}$ & .02 \\
\hline Median & 5.8 & 0 & & \\
\hline Range & $(0.0-29.5)$ & $(0.0-14.9)$ & & \\
\hline
\end{tabular}

$\mathrm{Cl}=$ confidence interval; $\mathrm{SD}=$ standard deviation; $\mathrm{GEE}=$ generalized estimating equation.

* Odds ratio from logistic regression in generalized linear model framework with GEEs.

† Odds ratio from multinomial regression in generalized linear mixed model.

₹ Difference based on parameter estimate from linear regression in generalized linear model framework with GEE. 
Each subject attempted to palpate the LHBT on the left side and right side on each of 3 models. Thus, each subject had 6 attempts before ultrasound training and 6 attempts after ultrasound training. During the pretraining period, the number of accurate palpations per subject ranged from 0 to 3 with a median of 1 . After ultrasound training, palpation accuracy improved to a median of 3 with a range of 1 to $6(P=.02)$. Of the 10 subjects, 8 improved their accuracy after ultrasound training, whereas 1 maintained the same level of accuracy and 1 did slightly worse (Figure 8).

\section{Discussion}

Previous studies demonstrated that the palpation accuracy of musculoskeletal structures is often poor [4-10]. This is the first study to our knowledge to evaluate the use of ultrasound as an educational tool to assist in correctly identifying the LHBT within the bicipital groove.

Our preinterventional accuracy rate was $20 \%$. This was greater than hypothesized based on a previous study palpating the same structure [4]. Given our small subject numbers, we were unable to define whether level of training was a significant factor in baseline accuracy. Further research is required to determine whether level of training or experience affects LHBT palpation accuracy.

The ultrasound intervention improved the palpation accuracy rate from $20 \%$ to $51.7 \%$, indicating that palpation attempts were 4.2 times more likely to be in the correct location after ultrasound training than at baseline $(P<.001)$. When looking at each subject individually, we found that 8 improved their accuracy after ultrasound training whereas 1 maintained the same level of accuracy and 1 did slightly worse (Figure 8).

Secondary study outcomes also demonstrated improvement after the ultrasound intervention. At baseline, our residents missed medially $36.7 \%$ of the time and laterally $43.3 \%$ of the time. These results

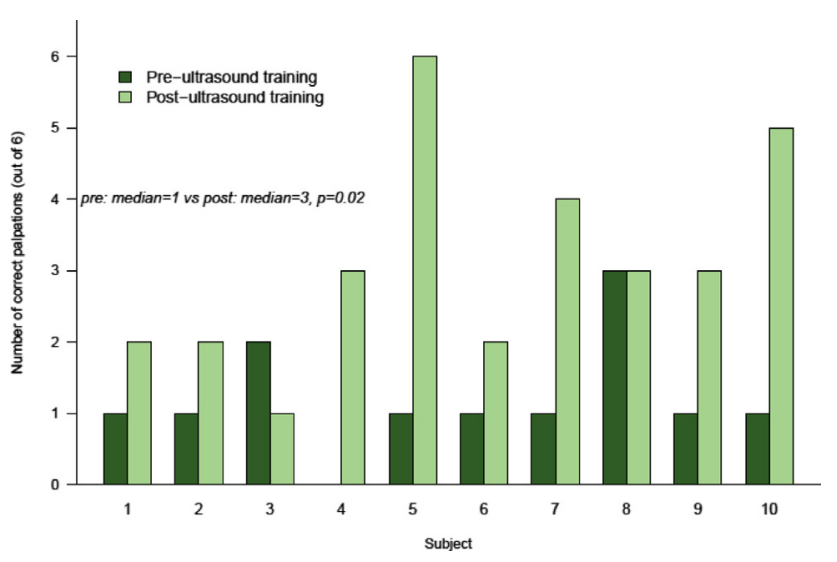

Figure 8. Number of correct palpations by subject pre- versus postintervention. differed compared with the previous study by Gazzillo et al [4], where all of the missed attempts were medial. However, when the distance and direction of the pretraining misses were averaged, our residents had a mean miss of $0.9 \mathrm{~mm}$ medially, which suggests that the magnitude of medial inaccuracy was greater than lateral inaccuracy. It is possible that the reason examiners tend to miss medially is because medical professionals are taught to identify the intertubercular groove by first palpating the greater tuberosity, then moving medially. Examiners may be mistaking the lesser tuberosity for the greater tuberosity during palpation, causing them to inaccurately identify the intertubercular groove medial to its correct location [4].

After the ultrasound training, the medial miss rate was drastically reduced as compared with the pretraining period $(8.3 \%$ versus $36.7 \%)$. This suggests that our training session was able to correct the previous tendency for incorrectly identifying the tendon medial to the groove through visual confirmation of the tendon location. After correcting the medial misses, we found that our mean miss distance and direction posttraining became $2.5 \mathrm{~mm}$ laterally. The lateral miss rate posttraining remained relatively stable compared with pretraining (40\% versus $43.3 \%)$. The mean absolute miss distance (ignoring direction) pre- versus post-training was $7.5 \mathrm{~mm}$ to $4.4 \mathrm{~mm}(P=.02)$ with an improved range from 0-29.5 $\mathrm{mm}$ to $0-14.9 \mathrm{~mm}$. Subjects' missed attempts were more accurate with a tightened range and less variability after the training session, indicating that the ultrasound training was helpful.

There were several limitations to this study that warrant discussion. First, the study had relatively few subjects and models, which did not allow for subgroup analysis (level of training, model body habitus, etc). One would assume that differences in body habitus would affect palpation accuracy and also may affect the efficacy of the ultrasound teaching intervention. It is also possible that level of training or experience may influence LHBT palpation accuracy. Both of these questions need to be investigated in future studies.

Second, although attempts were made to control for measurement errors, there is always the possibility that measurement errors occurred (ie, movement of subjects, the needle not being placed in exact spot as palpation attempt, ultrasound transducer not directly over the top of the needle/perpendicular to the floor, etc).

Lastly, the study includes the lack of a control group. Subjects were used as their own internal controls to assess baseline accuracy rates. Therefore, one cannot definitively conclude that the improvements in LHBT palpation accuracy were due to the ultrasound intervention rather than a learning affect from repetitively palpating the LHBT. Furthermore, because subjects were not brought back at a later date to evaluate their LHBT palpation accuracy, longevity of the LHBT 
palpation accuracy improvements could not be assessed in the current study. Further research with a control group and intermediate to long-term follow-up would help answer these questions.

\section{Conclusion}

This was the first study to evaluate the utility of ultrasound as an educational tool to improve the accuracy of correctly palpating the LHBT within the bicipital groove. Our findings demonstrate that palpation accuracy improves after ultrasound-assisted LHBT palpation training. These data suggest that the use of ultrasound may be beneficial when teaching musculoskeletal palpation skills to health care professionals.

\section{References}

1. Johnson JE, Carpenter JL. Medical house staff performance in physical examination. Arch Intern Med 1986;146:937-941.

2. York NL, Niehaus AH, Markwell SJ, Folse JR. Evaluation of students' physical examination skills during their surgery clerkship. Am J Surg 1999;177:240-243.

3. Simel DL. Time, now, to recover the fun in the physical examination rather than abandon it. Arch Intern Med 2006;166:603-604.

4. Gazzillo GP, Finnoff JT, Hall MM, Sayeed YA, Smith J. Accuracy of palpating the long head of the biceps tendon: An ultrasonographic study. PM R 2011;3:1035-1040.

5. Rho ME, Chu SK, Yang A, Hameed F, Lin CY, Hurh PJ. Resident accuracy of joint line palpation using ultrasound verification. PM R 2014;6:920-925.
6. Peck E, Lai JK, Pawlina W, Smith J. Accuracy of ultrasound-guided versus palpation-guided acromioclavicular joint injections: A cadaveric study. PM R 2010;2:817-821.

7. Scillia A, Issa K, Mclnerney VK, et al. Accuracy of in vivo palpationguided acromioclavicular joint injection assessed with contrast material and fluoroscopic evaluations. Skeletal Radiol 2015;44: 1135-1139.

8. Curtiss HM, Finnoff JT, Peck E, Hollman J, Muir J, Smith J. Accuracy of ultrasound-guided and palpation-guided knee injections by an experienced and less-experienced injector using a superolateral approach: A cadaveric study. PM R 2011;3:507-515.

9. Wisniewski SJ, Smith J, Patterson DG, Carmichael SW, Pawlina W. Ultrasound-guided versus nonguided tibiotalar joint and sinus tarsi injections: A cadaveric study. PM R 2010;2:277-281.

10. Karvelas K, Ziegler C, Rho ME. Resident accuracy of electromyography needle electrode placement using ultrasound verification. PM R 2016;8:748-753.

11. Hoppmann R, Hunt $\mathrm{P}$, Louis $\mathrm{H}$, et al. Medical student identification of knee effusion by ultrasound. ISRN Rheumatol 2011;2011:874596.

12. Shapiro RS, Ko PK, Jacobson S. A pilot project to study the use of ultrasonography for teaching physical examination to medical students. Comput Biol Med 2002;32:403-409.

13. Tshibwabwa ET, Groves HM, Levine MA. Teaching musculoskeletal ultrasound in the undergraduate medical curriculum. Med Educ 2007; 41:517-518.

14. Fodor D, Badea R, Poanta L, Dumitrascu DL, Buzoianu AD, Mircea PA. The use of ultrasonography in learning clinical examination - a pilot study involving third year medical students. Med Ultrason 2012;14:177-181.

15. Butter J, Grant TH, Egan M, et al. Does ultrasound training boost Year 1 medical student competence and confidence when learning abdominal examination? Med Educ 2007;41:843-848.

16. Ahn JS, French AJ, Thiessen ME, et al. Using ultrasound to enhance medical students' femoral vascular physical examination skills. J Ultrasound Med 2015;34:1771-1776.

\section{Disclosure}

R.W. Department of Physical Medicine and Rehabilitation, Mayo Clinic College of Medicine, Mayo Clinic, Rochester, MN

Disclosure: nothing to disclose

S.J.W. Department of Physical Medicine and Rehabilitation, Mayo Clinic College of Medicine, Mayo Clinic Sports Medicine Center, Rochester, MN

Disclosure: nothing to disclose

D.R.L. Department of Physical Medicine and Rehabilitation, University of Michigan, Ann Arbor, Ml

Disclosure: nothing to disclose

T.P.P. Department of Anesthesiology, Mayo Clinic College of Medicine, Mayo Clinic, Rochester, MN

Disclosure: nothing to disclose
D.R.L. Department of Health Sciences Research, Mayo Clinic College of Medicine, Mayo Clinic, Rochester, MN Disclosure: nothing to disclose

J.T.F. Department of Physical Medicine and Rehabilitation, Mayo Clinic College of Medicine, Mayo Clinic Sports Medicine Center, 200 First St SW, Rochester, MN 55905. Address correspondence to: J.T.F.; e-mail: Finnoff.Jonathan@mayo.edu Disclosure: nothing to disclose

Funding source: resident research funds Mayo Clinic Department of Physical Medicine and Rehabilitation.

Submitted for publication March 21, 2017; accepted November 29, 2017. 